\title{
Pool Boiling in Microgravity: Recent Results and Perspectives for the Project DEPA-SJ10
}

\author{
Shi-Xin Wan · Jian-Fu Zhao
}

Received: 24 February 2008 / Accepted: 9 May 2008 / Published online: 25 June 2008

(C) Springer Science + Business Media B.V. 2008

\begin{abstract}
Two research projects on pool boiling in microgravity have been conducted aboard the Chinese recoverable satellites. Ground-based experiments have also been performed both in normal gravity and in short-term microgravity in the Drop Tower Beijing. Steady boiling of R113 on thin platinum wires was studied with a temperature-controlled heating method, while quasi-steady boiling of FC-72 on a plane plate was investigated with an exponentially increasing heating voltage. In the first case, slight enhancement of heat transfer is observed in microgravity, while diminution is evident for high heat flux in the second one. Lateral motions of bubbles on the heaters are observed before their departure in microgravity. The surface oscillation of the merged bubbles due to lateral coalescence between adjacent bubbles drives it to detach from the heaters. The Marangoni effect on the bubble behavior is also discussed. The perspectives for a new project DEPA-SJ10, which has been planned to be flown aboard the Chinese recoverable satellite SJ-10 in the future, are also presented.
\end{abstract}

Keywords Microgravity $\cdot$ Pool boiling • Chinese recoverable satellite • Drop Tower Beijing • Review

Paper was submitted on the Third International Topical Team Workshop on TWO-PHASE SYSTEMS FOR GROUND AND SPACE APPLICATIONS September 10-12, 2008, Brussels, Belgium.

S.-X. Wan · J.-F. Zhao $(\varangle)$

National Microgravity Laboratory, Institute of Mechanics, Chinese Academy of Sciences,

15 Beisihuan Xilu, Beijing 100190, China

e-mail: jfzhao@imech.ac.cn

\section{Introduction}

Boiling is an extremely complicated and illusive process. Microgravity experiments offer a unique opportunity to study the complex interactions without external forces, such as buoyancy, which can affect the bubble dynamics and the related heat transfer. Furthermore, they can also provide a means to study the actual influence of gravity on the boiling. On the progress in this field, several comprehensive reviews, for example, Straub (2001), Di Marco (2003), Kim (2003), and Ohta (2003) among many others, are available.

In the past years, two research projects on pool boiling in microgravity have been conducted utilizing both the Chinese recoverable satellites and the Drop Tower Beijing in the National Microgravity Laboratory/CAS. Some of the research findings will be highlighted here. Furthermore, the perspectives are also presented here for a new project DEPA-SJ10 (bubble Dynamics and hEat transfer in Pool boiling on an artificial cAvity in microgravity), which has been planned to be flown aboard the Chinese recoverable satellite SJ-10 in the future.

\section{Experimental Facilities}

In the first project, a temperature-controlled pool boiling (TCPB) device has been developed (Liu 2006; Wan et al. 2003). The working liquid is degassed R113 at subcooled conditions. A platinum wire of $60 \mu \mathrm{m}$ in diameter and $30 \mathrm{~mm}$ in length is simultaneously used as heaters and thermometers. The heater resistance, and thus its temperature, is kept constant by a feedback circuit. There're 16 set-points in the range of the heater 
temperature from $35^{\circ} \mathrm{C}$ to $135^{\circ} \mathrm{C}$. The heater temperature is controlled stepping up-down-up in the experiments. According to Straub (2001), each step lasts about $30 \mathrm{~s}$ in order to obtain steady pool boiling. The TCPB device has been flown aboard the 22nd Chinese recoverable satellite RS-22 in September, 2005.

In the second project, a quasi-steady pool boiling (QSPB) device has been developed (Yan 2007; Zhao et al. 2007a). The working fluid is degassed FC-72. A plane plate heater with an effective heating area of $15 \times 15 \mathrm{~mm}^{2}$ is used. A quasi-steady heating method is adopted, in which the heating voltage is controlled as an exponential function with time, namely $U=$ $U_{0} \exp \left(\tau / \tau_{0}\right)$, where $\tau$ denotes the heating time. In order to make the heating process as a quasi-steady state, the parameter $\tau_{0}$ is set for $80 \mathrm{~s}$, which satisfies the quasisteady heating condition (Johnson 1971). The system pressure and the liquid subcooling are also controlled separately during the experimental runs. The QSPB device has been flown aboard the Chinese recoverable satellite SJ-8 in September, 2006.

\section{Experimental Results}

\section{Pool Boiling on Thin Wires}

Detailed experiment conditions of the space and ground-based experiments are listed in Table 1 (Liu 2006).

Figure 1 shows the heat transfer curves obtained in the flight experiment. It's found that the agreements between the prediction by Kuehn and Goldstein (1976) and the measured single-phase natural convection both in normal and in microgravity warrant reasonable confidence in the data. Following the single-phase natural convection, an explosive boiling occurs as soon as the heater temperature switches to the 16th set-point both in normal and in microgravity, and then transfer to two-mode transition boiling due to the effect of surface tension. Thus, the superheat at the onset of boiling is independent on gravity, or at least, dependent much weakly on gravity. In the nucleate boiling regime, heat

Table 1 Experimental conditions in the project TCPB-RS22

\begin{tabular}{lll}
\hline & $P(\mathrm{kPa})$ & $\Delta T_{\text {sub }}\left({ }^{\circ} \mathrm{C}\right)$ \\
\hline Ground exp. before flight & 101.2 & 25.5 \\
Space exp. & 101.0 & 26.2 \\
Ground exp. after flight & 101.4 & 27.1 \\
Drop tower exp. & 101.6 & 24.1 \\
\hline
\end{tabular}

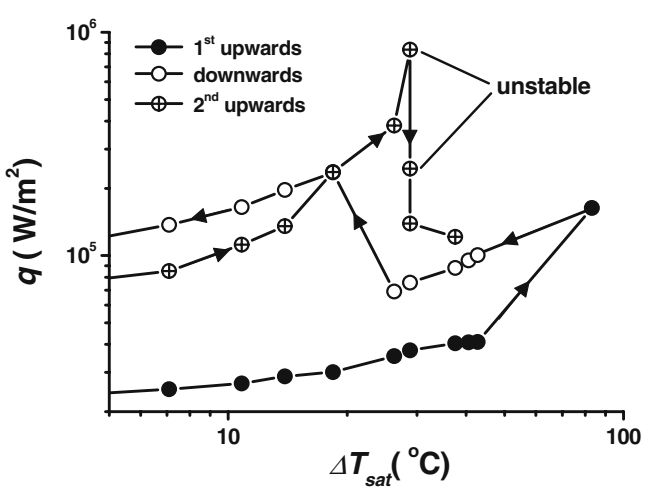

Fig. 1 Heat transfer curves from a thin wire in microgravity

transfer in microgravity is the same or slightly enhanced comparing with that in normal gravity (Fig. 2).

As shown in Fig. 3, it's also found that LienhardDhir-Zuber model (Lienhard and Dhir 1973; Zuber et al. 1961) can give a good prediction on the trend of $\mathrm{CHF}$ in different gravity conditions, although the value of the dimensionless radius $R^{\prime}=R_{\text {wire }} \sqrt{g\left(\rho_{L}-\rho_{V}\right) / \sigma}$ is far beyond its initial application range. Here, it ought to pointed out that due to no measurement for the residual gravity during the flight, it is estimated in the range of $\left(10^{-3} \sim 10^{-5}\right) g_{0}$ based on the previous flights of the same kind of satellites. These observations are consistent with Straub (2001). Furthermore, comparing the trend of CHF in Fig. 3 with the common viewpoint on the scaling of $\mathrm{CHF}$, it's inferred, as pointed out by Di Marco and Grassi (1999), that the dimensionless radius $R^{\prime}$, or the Bond number, may not be able to scale adequately the effects and to separate groups containing gravity due to complex competition of different mechanisms for small cylinder heaters.

A forward-and-backward lateral motion of vapor bubbles is observed along the wire before their departure in microgravity (Fig. 4) (Zhao et al. 2007b, 2007c).

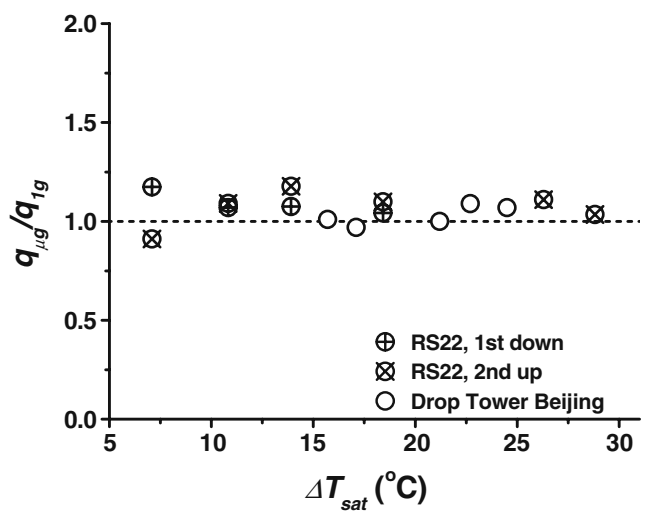

Fig. 2 Microgravity efficiency on heat transfer at nucleate boiling appropriate to Fig. 1 


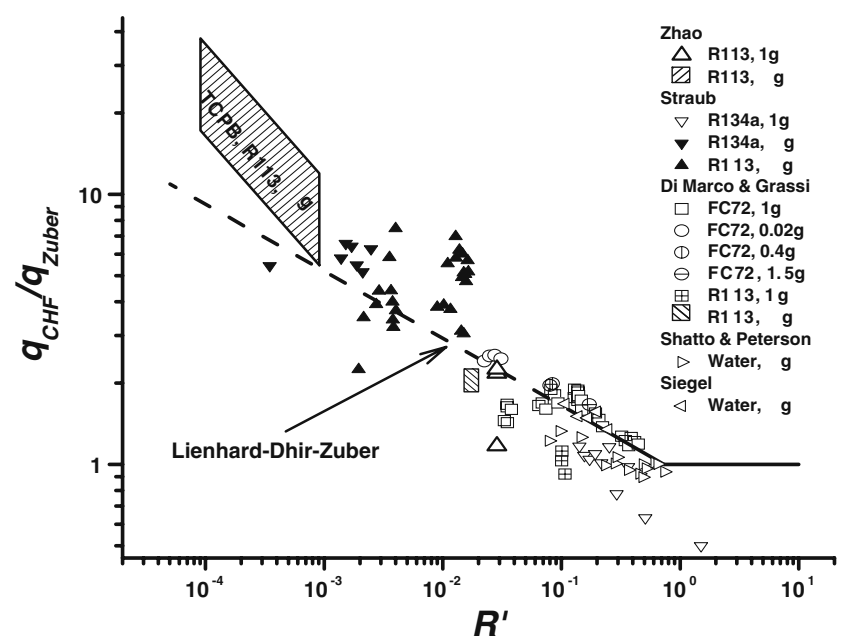

Fig. 3 Scaling of CHF with gravity in boiling on cylinders

Generally, the lateral velocity $U$ increases with the decrease of the bubble size, which can be interpreted by Marangoni convection around adjacent bubbles (Zhao et al. 2006). This kind of lateral motion can lead to lateral coalescence between adjacent bubbles. The surface of the merged bubble oscillates in this process, which may be caused by the interaction between the surface tension and vapor inertia. Then, the bubble will be lift up, namely detachment occurs. It's found that the oscillation due to coalescence of adjacent bubbles is the primary reason of bubble departure in the fully developed nucleate boiling regime in microgravity.

Three critical bubble diameters, namely $0.3 \mathrm{~mm}$, $3.5 \mathrm{~mm}$, and $8.4 \mathrm{~mm}$, are observed in the discrete vapor bubble regime in microgravity, which divide the observed vapor bubbles into four regions: Tiny bubbles are continually forming and growing on the surface before departing slowly from the wire when their sizes exceed the first critical value. The bigger bubbles, however, can be found staying on the surface again when their diameters are greater than the second critical

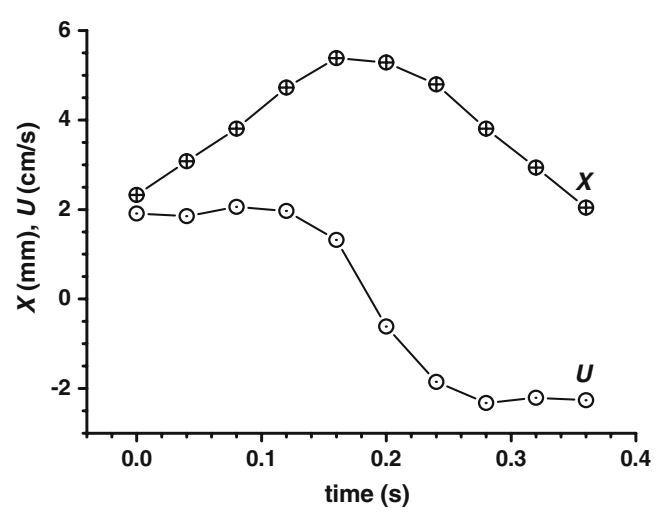

Fig. 4 Position $X$ and lateral velocity $U$ of a typical bubble

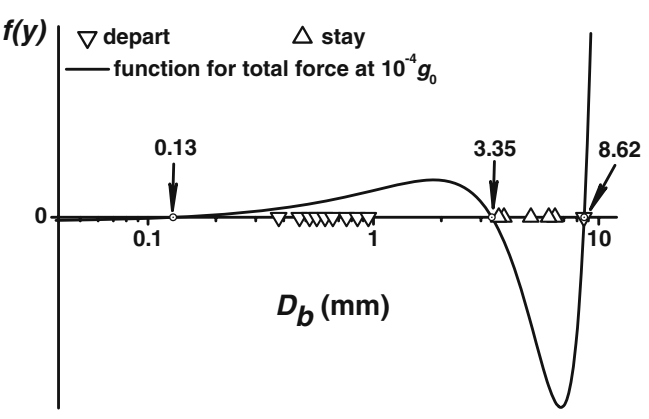

Fig. 5 Bubble departure diameters in microgravity

value. If they grow further bigger than the third critical, departure will be observed again. The first two kinds of bubbles can be observed both in normal and in microgravity conditions, while the others only in microgravity. In the traditional bubble departure models, no one can predict the whole observation. Based on the model of Lee (1992), a qualitative model is proposed (Zhao et al. 2008), in which the Marangoni effect is taken into account. In normal gravity, the function for the total forces acting on the growing bubble, $f(y)$, has only one zero-value point, indicting only one critical diameter for bubble departure. But, the second and third zerovalue points will be predicted by the new model when the gravity reduces to no more than $1.36 \times 10^{-4} g_{0}$, indicting three critical diameters existing for bubble departure which can divide the whole range of the observed bubbles into four regimes as the observation in microgravity. Figure 5 shows the comparison between the prediction and the observation. The agreement is quite evident.

\section{Pool Boiling on Plane Plate}

There are 8 runs obtained in the space experiment. The experimental conditions are shown in Fig. 6, which can be classified into four groups according to dif-

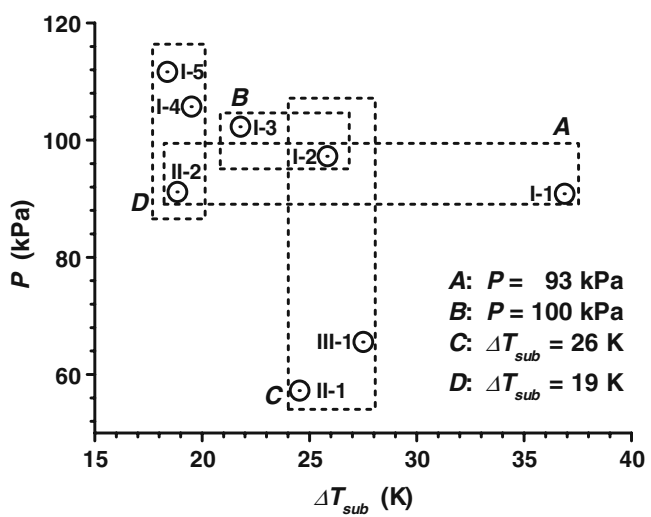

Fig. 6 Experimental conditions in the project QSPB-SJ8 


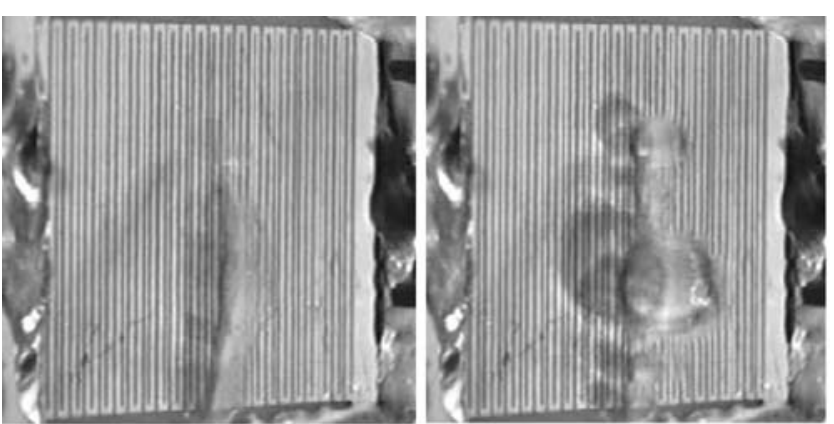

(a)

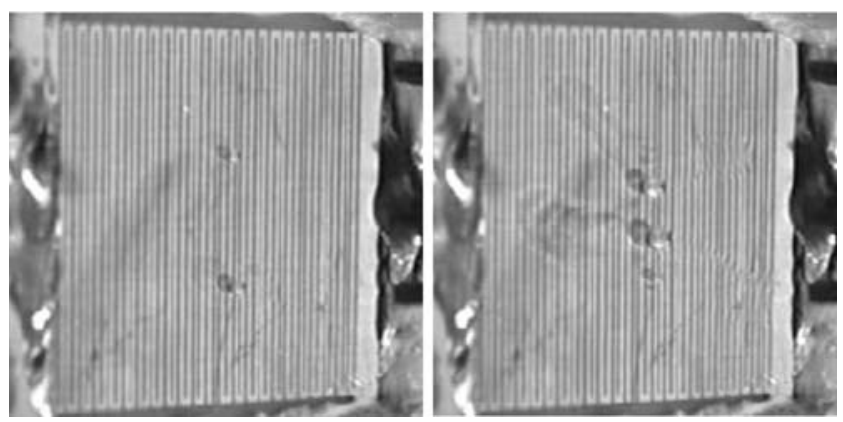

(b)

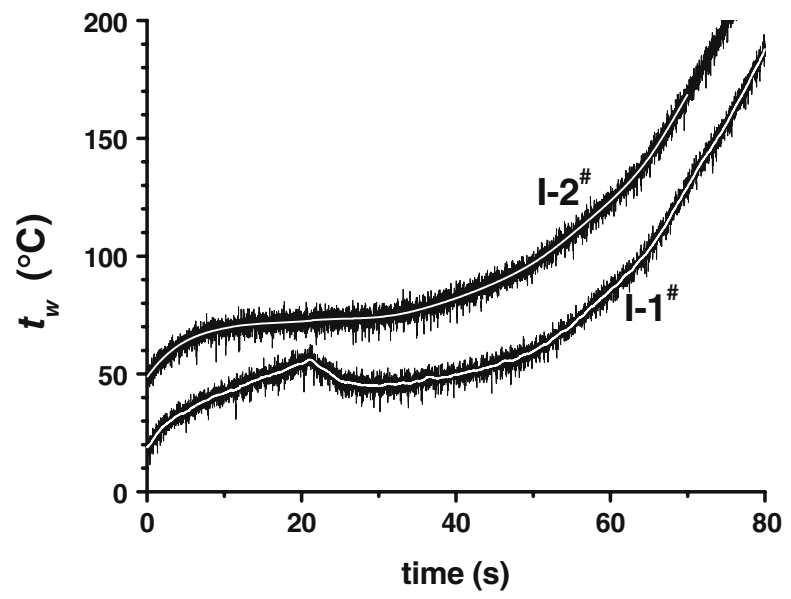

(c)

Fig. 7 The onset of boiling in microgravity. a Explosive boiling in the first run. b Bubbles grown gradually in the second run. c Heater temperature in the 1 st and 2 nd runs

ferent pressure and/or subcooling (Yan 2007; Zhao et al. 2007a). Before the space flight, several ground experiments have performed. The results are in good agreement with those of Honda et al. (2002) and Rainey et al. (2003), which warrants reasonable confidence in the data.

In the first run, the first appearance of bubbles is abrupt and explosive (Fig. 7a), and an obvious overshooting is observed in the heater temperature, correspondingly (Fig. 7c). In the following runs, the bubble

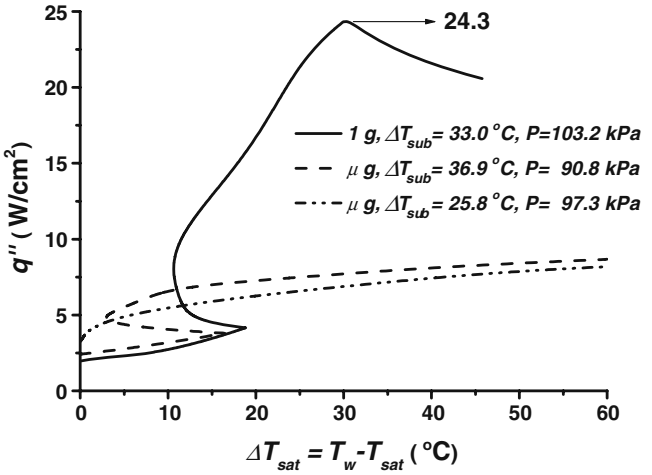

Fig. 8 Boiling curves on plate in different gravity

is observed to grow slowly and even at an obvious standstill after their first appearance (Fig. 7b shows an example in the second run). Correspondingly, no overshooting can be observed in the heater temperature (Fig. 7c. Here the curve for the second run has been shifted $15^{\circ} \mathrm{C}$ up for the reason of clarity). It may indicate that there could be residual micro-bubbles in cavities after the preceding runs, which make the cavities easier to be activated.

In Fig. 8, the boiling curves in microgravity are compared with the control experimental result in normal gravity. The boiling curves in microgravity have much smaller slopes than those in normal gravity, which is consistent with previous results, for example, Lee et al. (1997) among others. Furthermore, the boiling curves in microgravity even have no turning point, resulting in some difficulties in determining accurately the occurrence of CHF. It might be caused by the side effects due to the limited heater size, although no clear observation has made on the local motion structure adjacent to the heater surface near CHF since the CCD camera recorded images only from a single direction at about $45^{\circ}$ with the heater surface.

For the same pressure, nucleate boiling occurred significantly earlier at a quite lower wall superheat, and thus more efficient of heat transfer in low heat flux can be observed in microgravity. In high heat flux, however, much evident diminution of heat transfer can be observed and the values of CHF are also quite lower in microgravity, which is no more than $40 \%$ of that in terrestrial condition.

It's found that the nucleate boiling will be enhanced with the increase of subcooling or pressure for the same pressure or subcooling in microgravity, and that CHF will also increase with the subcooling and/or pressure. These characteristics are similar with those observed on Earth.

It's also observed that vapor bubbles glide on the heating surface throughout the boiling process. They 
will collide with each other and coalesce to form the bigger coalesced bubble. The coalesced bubble absorbs continuously the primary bubbles until it covers the whole heater surface. The growing velocity of the coalesced bubble decreases with the increase of subcooling, since the condensation at the top of bubbles will increase (Li et al. 2008).

\section{Perspectives for the Project DEPA-SJ10}

A new project DEPA-SJ10 has been planned to be flown aboard the Chinese recoverable satellite SJ-10 in the near future. This project is a part of the program of the SJ-10 recoverable space scientific experiment satellite for microgravity and space life science research, which is one of the Chinese major space activities in the 11th 5-year plan (2007-2012) and scheduled to be launched in 2010.

In the project DEPA-SJ10, a series of single bubble experiments will be used as a model of subsystems in nucleate pool boiling of pure substances. Figure 9 shows briefly the schematic of the heater, the boiling chamber and the visualization of the bubble behaviors. Carefully degassed FC-72 will be used as the working fluid. A special designed plane heater with an artificial cavity will be used to produce a single vapor bubble at fixed location. Transient processes of bubble formation, growth and detachment will be observed, while the temperature distribution near the active nucleation site will be measured at subcooling and saturated conditions. The main aim is to describe bubble behavior and convection around the growing vapor bubble in microgravity, to understand small scale heat transfer mechanisms, and to reveal the physical phenomena governing nucleate boiling.

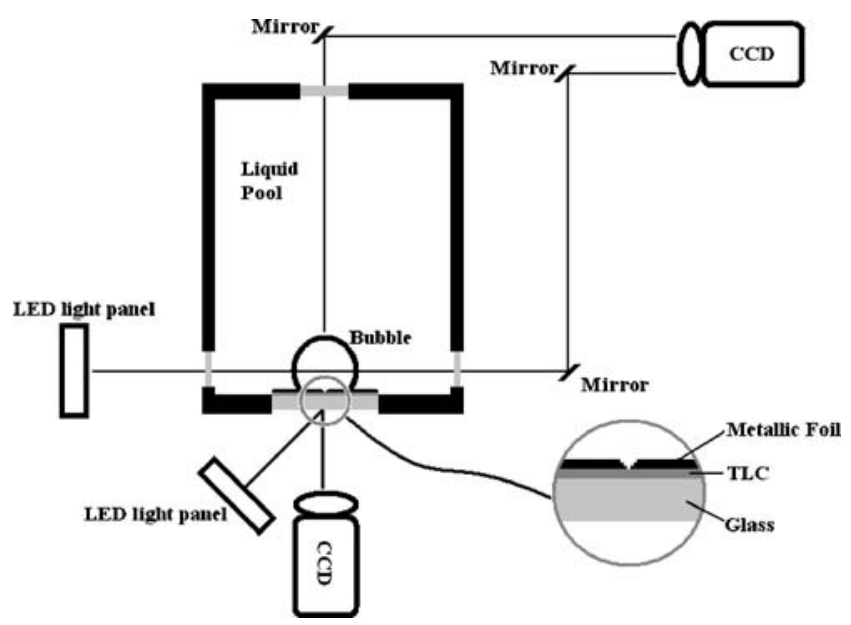

Fig. 9 Schematic of the heater and visualization

\section{Conclusion}

Utilizing the Chinese recoverable satellites, we have conducted two projects on study of pool boiling heat transfer in microgravity. Ground-based experiments have also been performed both in normal gravity and in short-term microgravity in the Drop Tower Beijing. Two kinds of heater are used. One is a thin platinum wire, the other is a plane plate. Steady pool boiling of R113 on the wire with a temperature-controlled heating method is studied, while quasi-steady pool boiling of FC-72 on the plate heater with an exponentially increasing heating voltage is studied. It's found that only slight enhancement of heat transfer can be observed for the wire case, while much large diminution can be observed in high heat flux for the plate case. The bubble patterns are dramatically altered by the variation of the acceleration in both cases. Lateral motions of vapor bubbles were observed before their departure in microgravity. Generally, the lateral velocity increases with the decrease of the bubble size. This kind of lateral motion can lead to lateral coalescence between adjacent bubbles. The surface of the merged bubble oscillates in this process and then the bubble will be lift up, namely detachment occurs. The Marangoni effect may play an important role in the discrete bubble dynamic behaviors. Further study on the origin of the Marangoni effect in a liquid-vapor system with phase change is needed.

A new project, DEPA-SJ10, has also been planned to be flown in the near future. Some major features are described briefly, and the perspectives for this project are also presented.

Acknowledgements The studies presented here are supported financially by the National Natural Science Foundation of China (10432060), and the Chinese Academy of Sciences (KACX2-SW02-03, KJCX2-SW-L05). The authors greatly appreciate Prof. W. R. Hu (NMLC, China), Prof. H. Ohta (Kyushu University, Japan), Prof. L. Xi and Prof. J. Su (AOE/CAS, China), and Prof. H. X. Li (Xi'an Jiaotong University, China) for their contributions to the projects. The authors are also very grateful for the outstanding work of our graduate students: G. Liu, N. Yan, Z. D. Li, J. Li, and Y. H. Lu.

\section{References}

Di Marco, P.: Review of reduced gravity boiling heat transfer: European research. J. Jpn. Microgravity Appl. 20, 252-263 (2003)

Di Marco, P., Grassi, W.: About the scaling of critical heat flux with gravity acceleration in pool boiling. Proc. XVII UIT Nat. Heat Transfer Conf., Ferrara, pp. 139-149 (1999)

Honda, H., Takamastu, H., Wei, J.J.: Enhanced boiling of FC72 on Silicon chips with micro-pin-fins and submicron-scale 
roughness. J. Heat Transfer. Trans. ASME. 124(2), 383-390 (2002)

Johnson, H.A.: Transient boiling heat transfer to water. Int. J. Heat Mass Transfer. 14, 67-82 (1971)

Kim, J.: Review of reduced gravity boiling heat transfer: US research. J. Jpn. Microgravity Appl. 20, 264-271 (2003)

Kuehn, T.H., Goldstein, R.J.: Correlating equations for natural convection heat transfer between horizontal circular cylinders. Int. J. Heat Mass Transfer. 19, 1127-1134 (1976)

Lee, D.J.: Bubble departure radius under microgravity. Chem. Eng. Comm. 117, 175-189 (1992)

Lee, H.S., Merte, H. Jr., Chiaramonte, F.: Pool boiling curve in microgravity. J. Thermophy. Heat Transfer. 11(2), 216-222 (1997)

Li, J., Zhao, J.F., Yan, N., Li, Z.D., Ohta, H.: Bubble behavior in microgravity pool boiling. J. Eng. Thermophy. 29, 439-442 (2008)

Lienhard, J.H., Dhir, V.K.: Hydrodynamic prediction of peak pool boiling heat fluxes from finite bodies. J. Heat Transfer. Trans. ASME. 95, 152-158 (1973)

Liu, G.: Study of subcooled pool boiling heat transfer on thin platinum wires in different gravity conditions. M. Sc. Thesis, Institute of Mechanics, Chinese Academy of Sciences, Beijing, China (2006)

Ohta, H.: Review of reduced gravity boiling heat transfer: Japanese research. J. Jpn. Microgravity Appl. 20, 272-285 (2003)

Rainey, K.N., You, S.M., Lee, S.: Effect of pressure, subcooling, and dissolved gas on pool boiling heat transfer from microporous surfaces in FC-72. J. Heat Transfer, Trans. ASME. 125, 75-83 (2003)
Straub, J.: Boiling heat transfer and bubble dynamics in microgravity. Adv. Heat Transfer. 35, 57-172 (2001)

Wan, S.X., Zhao, J.F., Liu, G., Hu, W.R.: TCPB device: description and preliminary ground experimental results. In: 54th International Astronautical Congress, Bremen, Germany, September 29-October 3 (2003)

Yan, N.: Experimental study on pool boiling heat transfer in microgravity. M. Sc. Thesis, Institute of Mechanics, Chinese Academy of Sciences, Beijing, China (2007)

Zhao, J.F., Wan, S.X., Liu, G., Hu, W.R.: Experimental study on subcooled pool boiling in microgravity utilizing Drop Tower Beijing/NMLC. In: Proc. 5th Int. Symp. Multiphase Flow, Heat Mass Transfer \& Energy Conversion, vol. 4, pp. 1730$1735(2006)$

Zhao, J.F., Yan, N., Li, J., Li, Z.D., Hu, W.R., Ohta, H.: Pool Boiling Heat Transfer in Microgravity. In: 3rd International Symposium on Physical Sciences in Space, October 22-26, Nara, Japan (2007a)

Zhao, J.F., Liu, G., Li, Z.D., Wan, S.X.: Bubble Behaviors in Nucleate Pool Boiling on Thin Wires in Microgravity. In: 6th International Conference on Multiphase Flow, July 913, Leipzig, Germany (2007b)

Zhao, J.F., Wan, S.X., Liu, G., Li, Z.D., Hu, W.R.: Pool boiling heat transfer in microgravity. Microgravity Sci. Tech. 21(3/4), 135-136 (2007c)

Zhao, J.F., Liu, G., Wan, S.X., Yan, N.: Bubble dynamics in nucleate pool boiling on thin wires in microgravity. Microgravity Sci. Tech. (2008). doi:10.1007/s12217-008-9010-y

Zuber, N., Tribus, M., Westwater, J.W.: The hydrodynamic crisis of pool boiling of saturated and subcooled liquids. ASME Inter. Development in Heat Trans. 27, 230-236 (1961) 